This item was submitted to Loughborough's Research Repository by the author.

Items in Figshare are protected by copyright, with all rights reserved, unless otherwise indicated.

\title{
Using mass spectrometry to transform the assessment of sexual assault evidence
}

PLEASE CITE THE PUBLISHED VERSION

https://doi.org/10.1016/j.forc.2020.100262

PUBLISHER

Elsevier BV

VERSION

AM (Accepted Manuscript)

PUBLISHER STATEMENT

This paper was accepted for publication in the journal Forensic Chemistry and the definitive published version is available at https://doi.org/10.1016/j.forc.2020.100262.

\section{LICENCE}

CC BY-NC-ND 4.0

\section{REPOSITORY RECORD}

Rankin-Turner, Stephanie, Paul Kelly, Roberto SP King, and Jim Reynolds. 2020. "Using Mass Spectrometry to Transform the Assessment of Sexual Assault Evidence". Loughborough University. https://hdl.handle.net/2134/12628172.v1. 


\title{
Using mass spectrometry to transform the assessment of sexual assault evidence
}

\author{
Stephanie Rankin-Turner ${ }^{\mathrm{a}^{*}}$, Paul F. Kelly ${ }^{\mathrm{a}}$, Roberto S. P. King ${ }^{\mathrm{b}}$, James C. Reynolds ${ }^{\mathrm{a}^{*}}$ \\ ${ }^{a}$ Department of Chemistry, Loughborough University, Loughborough, Leicestershire, LE11 \\ 3TU, UK \\ ${ }^{\mathrm{b}}$ Foster + Freeman Ltd, Vale Park, Evesham, Worcestershire, WR11 1TD, UK \\ Corresponding author: J.C.Reynolds@lboro.ac.uk
}

\begin{abstract}
The time-consuming analysis of sexual assault evidence creates a challenging bottleneck in forensic investigations. Common techniques for semen analysis result in sample destruction, and there are currently no confirmatory techniques for the preparation-free or in situ analysis of semen. In all, there is a crucial requirement for the development of faster direct analytical techniques. In this study, the first use of ambient ionisation mass spectrometry for the forensic analysis of human semen has been demonstrated. Sheath-flow probe electrospray ionisation mass spectrometry (sfPESI-MS) was applied to the analysis of semen for the purpose of rapid body fluid identification, in addition to determining the effects of sample age on detection. Fresh and aged semen were readily detected from numerous surfaces, with a preparation-free analysis time of $<10$ seconds. The technique was also applied to the analysis of different types of condom, demonstrating the ability to detect differences in sheath and lubricant compositions, essential evidence in sexual assault investigations. This demonstrates the potential to both indicate prophylactic use and ascertain information regarding the condom used.
\end{abstract}

Keywords: Mass Spectrometry, Ambient Ionization, Analytical Methods, Forensic Chemistry, Body Fluids, Surface Analysis

\section{Introduction}

Over 150,000 sexual assaults are recorded by the police in England and Wales each year (159,740 during 2018),[1] though this is believed to be only $\sim 15 \%$ of actual incidents.[2] During the investigation of a sexual assault, biological evidence, particularly semen, is of great 
importance. The detection of semen at a crime scene, or on the victim's body, can provide essential information in both corroborating a victim's allegations, along with identifying the perpetrator through DNA analysis, often crucial for conviction. However, the rapid and reliable identification of suspected semen stains is not necessarily a straightforward process.

Various tests have been developed for the identification of semen, though the most popular and widely used is the acid phosphatase (AP) test. The enzyme acid phosphatase is produced by the prostate gland and as a result is present in particularly high concentrations in seminal fluid,[3] thus has become the obvious target for semen presumptive tests. Acid phosphatase catalyses the hydrolysis of organic phosphates, resulting in the formation of a product that will produce a coloured precipitate upon reacting with a diazonium salt, such as Brentamine Fast Blue A.[4] Unfortunately, many presumptive tests are non-specific, potentially reacting (false)positively with other materials, [3] and are, typically, destructive. Confirmatory tests for semen have been developed, such as the rapid stain identification (RSID) test for semen. This lateral flow immunochromatographic test strip detects the presence of human semenogelin, a protein produced in the seminal vesicles and thus abundant in semen.[5] However, RSID tests are single-use test strips that are also destructive to the sample, requiring sample extraction and application directly to the test strip itself. There is a need for the development of preparationfree, in situ semen analysis techniques. Furthermore, chemical analysis in the context of a sexual assault investigation is not limited to biological material. In recent years, the potential chemical information that can be obtained from condoms and sexual lubricants has become apparent, highlighting the need for analytical techniques which enable the detection of residues from prophylactics.

Ambient ionisation mass spectrometry enables the rapid, in situ analysis of samples in their native state, without the need for time-consuming and destructive sample preparation. Although mass spectrometry has been previously utilized in semen analysis, primarily for clinical metabolomics purposes, these studies typically utilize time-consuming, destructive and laboratory-confined techniques, such as gas and liquid chromatography mass spectrometry.[6]-[11] Despite the obvious advantages of ambient ionisation techniques, and the previous successful application of MS to direct biofluid analysis,[12]-[14] they have not yet been utilized in the analysis of semen.

Sheath-flow probe electrospray ionisation mass spectrometry (sfPESI-MS) is an ambient ionisation mass spectrometry technique developed by Hiraoka, et al.[15], [16] In brief, the sfPESI probe simply consists of a needle housed within a plastic solvent-filled sheath (Figure 
1). The base of the probe is briefly touched to the sample surface where analyte extraction occurs, before the application of a high voltage in front of the mass spectrometer inlet to induce electrospray ionisation. The probe is cheap to construct and enables the material to be sampled, ionized and analysed in less than ten seconds. The cheap construction of the probe means that it could be produced as a single-use consumable, eliminating concerns of cross-contamination. Previous research has demonstrated the effectiveness of sfPESI-MS in the analysis of biological materials,[17] but it has not yet been applied to the analysis of seminal fluid. This paper demonstrates the first application of ambient ionisation mass spectrometry to the forensic analysis of human semen.

\section{Materials and Methods}

\section{1 sfPESI Probe and Sampling Procedure}

The sheath-flow probe electrospray ionisation probe was composed of a $20 \mu 1$ gel-loading tip (epT.I.P.S, Eppendorf, Hamburg, Germany) with a $0.12 \mathrm{~mm}$ o.d stainless steel acupuncture needle (Seirin, Shizuoka, Japan) inserted. The needle protruded from the base of the sheath by approximately $0.1 \mathrm{~mm}$ and was held in place by a silicone septum at the top of the gel-loading tip. The sheath was filled with a water/ethanol solution $(50: 50 \mathrm{v} / \mathrm{v})$ containing $0.1 \mu \mathrm{g} / \mathrm{mL}$ caffeine. 99.5\% ethanol was purchased from Sigma-Aldrich (Gillingham, UK) and HPLC grade water was purchased from VWR (Lutterworth, UK).

When sampling, the base of the probe was touched to the surface of the sample for 5 seconds to enable analyte extraction, during which time the probe was held at ground potential. After extraction, the probe was position in front of the mass spectrometer inlet, approximately $2 \mathrm{~mm}$ above and in front of the inlet. A high voltage of $2.4 \mathrm{kV}$ was applied to the needle using a $2.5 \mathrm{kV}$ photomultiplier power supply (Brandenburg, 476R model) to induce electrospray. 

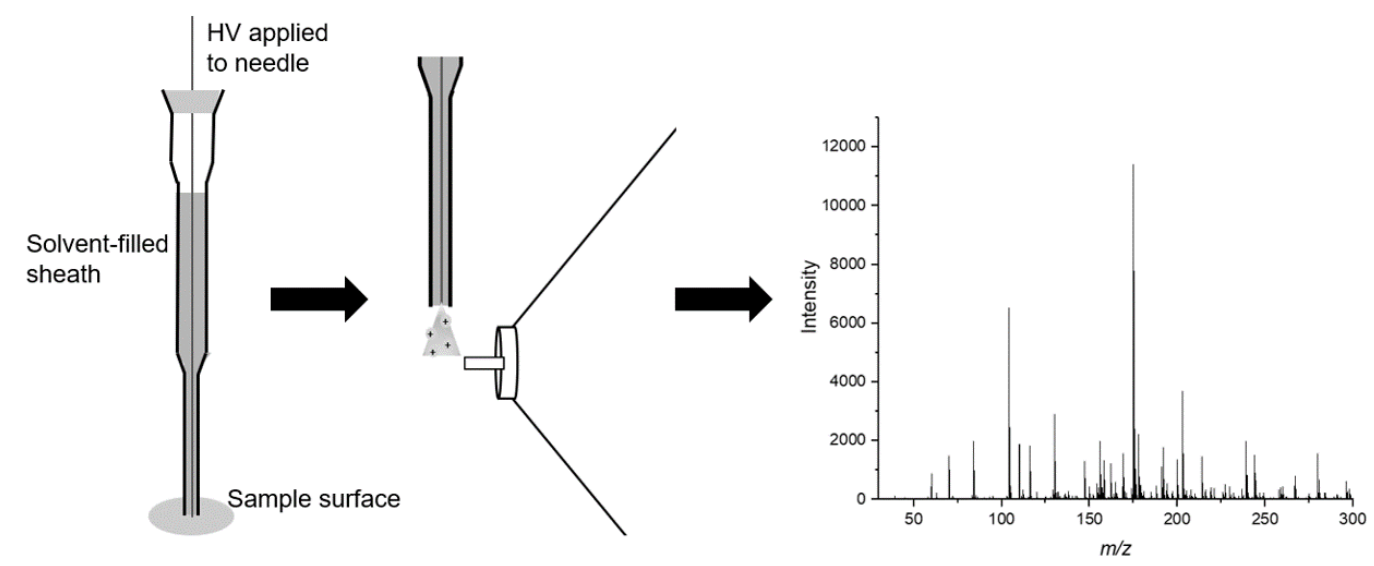

Figure 1. sfPESI schematic. A solvent-filled sheath holding a needle is briefly touched to the sample surface for analyte extraction. The probe is raised in front of the MS inlet and a high voltage is applied to induce electrospray for rapid mass spectra.

In this study, the sfPESI probe was coupled with a Waters Synapt mass spectrometer and applied to the analysis of human semen and condoms to demonstrate a new technique for the direct analysis of sexual assault evidence. In the analysis of semen, an anonymized, pooled semen sample (purchased from BioIVT) was applied in $5 \mu \mathrm{L}$ aliquots to glass slides, cotton fabric and swatches of Durex Featherlite condom (applied to the inside surface of the condom). Samples were either analysed immediately or stored under ambient conditions for ageing purposes. sfPESI-MS was directly applied to fresh and aged semen on a range of surfaces to assess the ability of the technique in semen detection. Compound identification was achieved using tandem mass spectrometry to induce ion fragmentation before comparison with online fragment ion databases.[18]

\subsection{Mass Spectrometry}

The sfPESI probe was coupled with a Waters Synapt high definition mass spectrometer. Experiments were performed in positive ion mode under the following conditions: sampling cone voltage $20 \mathrm{~V}$, extraction cone voltage $3 \mathrm{~V}$, source temperature $120^{\circ} \mathrm{C}$, transfer collision energy of $3 \mathrm{~V}$ and trap collision energy varied between 5 and $20 \mathrm{~V}$. Protonated caffeine was used as a lock mass throughout sample analyses. Data were acquired and analysed using MassLynx version 4.1 software.

\section{Results and Discussion}




\subsection{Semen Analysis}

Amino acids were found to be the most abundant compounds detected in semen by this technique, particularly proline, histidine, arginine and glutamine, which were detected as $[\mathrm{M}+\mathrm{H}]^{+}$and $[\mathrm{M}+\mathrm{Na}]^{+}$ions. In addition to these amino acids, spermine, choline, pyroglutamic acid, 3-hydroxysuberic acid, and glycerophosphocholine were also detected. A full list of compounds detected and identified, along with characteristic fragment ions, can be found in Table 1. It is important to note that in complex mixtures such as biological fluids, it is possible that multiple isobaric compounds are present at a given $\mathrm{m} / \mathrm{z}$ ratio, potentially complicating MS/MS spectra and making analyte identification difficult. Some compounds were not sufficiently abundant to isolate for tandem MS experiments, thus fragmentation could not be achieved for a number of ions of interest. For example, fragmentation ions could not be observed for components of interest detected at $\mathrm{m} / \mathrm{z}$ 60, 120 and 131, however based on previous research these most likely correspond to protonated triethylamine,[11] homoserine[11] and either citraconic acid or glutaconic acid[8] respectively, all of which are known components in seminal fluid.

Table 1. Fragmentation data for compounds of interest detected in human semen by sfPESI-MS/MS.

\begin{tabular}{|c|c|c|c|}
\hline Identification & $m / z$ & Formula & Fragment Ions \\
\hline Choline & 104.1 & $\mathrm{C}_{5} \mathrm{H}_{14} \mathrm{NO}$ & $45,58,60,86,104$ \\
\hline Proline & 116.1 & $\mathrm{C}_{5} \mathrm{H}_{10} \mathrm{NO}_{2}$ & 70,116 \\
\hline Dihydrothymine & 129.1 & $\mathrm{C}_{5} \mathrm{H}_{9} \mathrm{~N}_{2} \mathrm{O}_{2}$ & $69,73,84,112,129$ \\
\hline Pyroglutamic acid & 130.1 & $\mathrm{C}_{5} \mathrm{H}_{8} \mathrm{NO}_{3}$ & $44,56,84,130$ \\
\hline Leucine & 132.1 & $\mathrm{C}_{6} \mathrm{H}_{14} \mathrm{NO}_{2}$ & $44,86,132$ \\
\hline Glutamine & 147.1 & $\mathrm{C}_{5} \mathrm{H}_{11} \mathrm{~N}_{2} \mathrm{O}_{3}$ & $56,84,101,130,147$ \\
\hline Histidine & 156.1 & $\mathrm{C}_{6} \mathrm{H}_{10} \mathrm{~N}_{3} \mathrm{O}_{2}$ & $56,83,93,110,156$ \\
\hline Carnitine & 162.1 & $\mathrm{C}_{7} \mathrm{H}_{16} \mathrm{NO}_{3}$ & $60,85,102,103,120$ \\
\hline Arginine & 175.1 & $\mathrm{C}_{6} \mathrm{H}_{15} \mathrm{~N}_{4} \mathrm{O}_{2}$ & $\begin{array}{c}60,70,112,116,130,158 \\
175\end{array}$ \\
\hline 3-Hydroxysuberic acid & 191.1 & $\mathrm{C}_{8} \mathrm{H}_{15} \mathrm{O}_{5}$ & $67,109,155,173,191$ \\
\hline Spermine & 203.1 & $\mathrm{C}_{10} \mathrm{H}_{27} \mathrm{~N}_{4}$ & $58,72,84,112,129,203$ \\
\hline
\end{tabular}


A number of compounds were detected that are of particular relevance in the identification of semen, such as spermine. Spermine was detected as the $[\mathrm{M}+\mathrm{H}]^{+}$ion $\mathrm{m} / \mathrm{z} 203$ and its identity confirmed by the characteristic fragment ions $m / z 58,72,84,112,129$ (Figure S1). This biogenic amine is particularly abundant in semen and responsible for the characteristic odour of this body fluid.

Another abundant, and pertinent, compound found in semen, was choline, detected at $\mathrm{m} / z 104$ and identified based on characteristic fragment ions at $\mathrm{m} / z$ 45, 58 and 60 (Figure S1). Choline is an essential nutrient known to be abundant in seminal fluid and has previously been the target of forensic presumptive tests for semen. The Florence Iodine test detects choline by utilising an iodine-based reagent to produce brown choline periodide crystals, the amount of which is related to the concentration of choline.[19] Although compounds such as spermine and choline have been previously detected in other body fluids, the reported concentration has been relatively low in comparison to semen. Thus, the presence and relative abundance of these compounds, as detected by sfPESI-MS, could be used to rapidly confirm the presence of semen. The simultaneous detection of multiple components present in semen provides specificity far greater than standard presumptive tests can achieve, typically detecting only one component. Although these analytes have been detected in other bodily fluids, components such as spermine and choline are more abundant in semen, and previous analysis of body fluids by sfPESI has demonstrated different body fluids produce distinct mass spectra.[17] Urine was dominated by urea and creatinine, blood displayed strong signals for glucose/fructose, along with sodiated lipids, whereas saliva exhibited a complex mixture of low abundance acids. The distinct mass spectra obtained from these forensically-relevant body fluids highlights the specificity of this technique for the differentiation of semen from other biological fluids.

From a forensic perspective, in situ semen analysis requires the ability to apply the technique to samples that may be present on various substrate materials. In this study, both fresh and aged semen was sampled from cotton fabric, condom material, and glass. Sampling from fabric and condoms was performed to assess the ability to detect semen on two materials likely to be encountered during sexual assault casework, and glass was used to enable consistent and reliable analysis and ageing on a non-contaminated, non-porous surface. Fresh semen could be readily sampled and detected on all three surfaces assessed (Figure S2), with 
minimal differences observed in the overall profile of the most abundant components (i.e. the series of amino acids). Some differences were observed in the ratio of particular components. For instance, in the case of semen sampled from a glass surface, choline was observed at a greater intensity than all other components, whereas on the condom and fabric substrates, arginine was the most abundant compound detected. This is likely due to differences in surface porosity and surface interactions affecting the extent to which different chemical components are directly extracted from the sample.

The analysis of samples from highly contaminated surfaces by ambient ionisation MS can often cause problems with ion suppression due to the abundance of background contaminants and interferents. This could be particularly problematic in the case of sampling directly from prophylactic evidence types, which are coated with lubricants and other additives. When sfPESI was applied to semen samples on condoms, however, this issue was not encountered. The application of the sfPESI probe directly to the condom, afforded a mass spectrum that was dominated by prominent ions detected at $\mathrm{m} / z 130$ and 239 (Figure S3). However, when the probe was applied to fresh or dried semen on condom material, the sfPESI technique was capable of only detecting components originating from the semen sample, experiencing no background interference from the condom or lubricant. This is because the fine tip of the sfPESI probe could be used to target a specified area of approximately $1 \mathrm{~mm}^{2}$ (i.e. the body fluid stain only), thus allowing analyte extraction primarily from the semen stain itself. This demonstrates the power of the sfPESI probe in sampling target analytes even in the presence of a highly contaminated or complex background surface. 

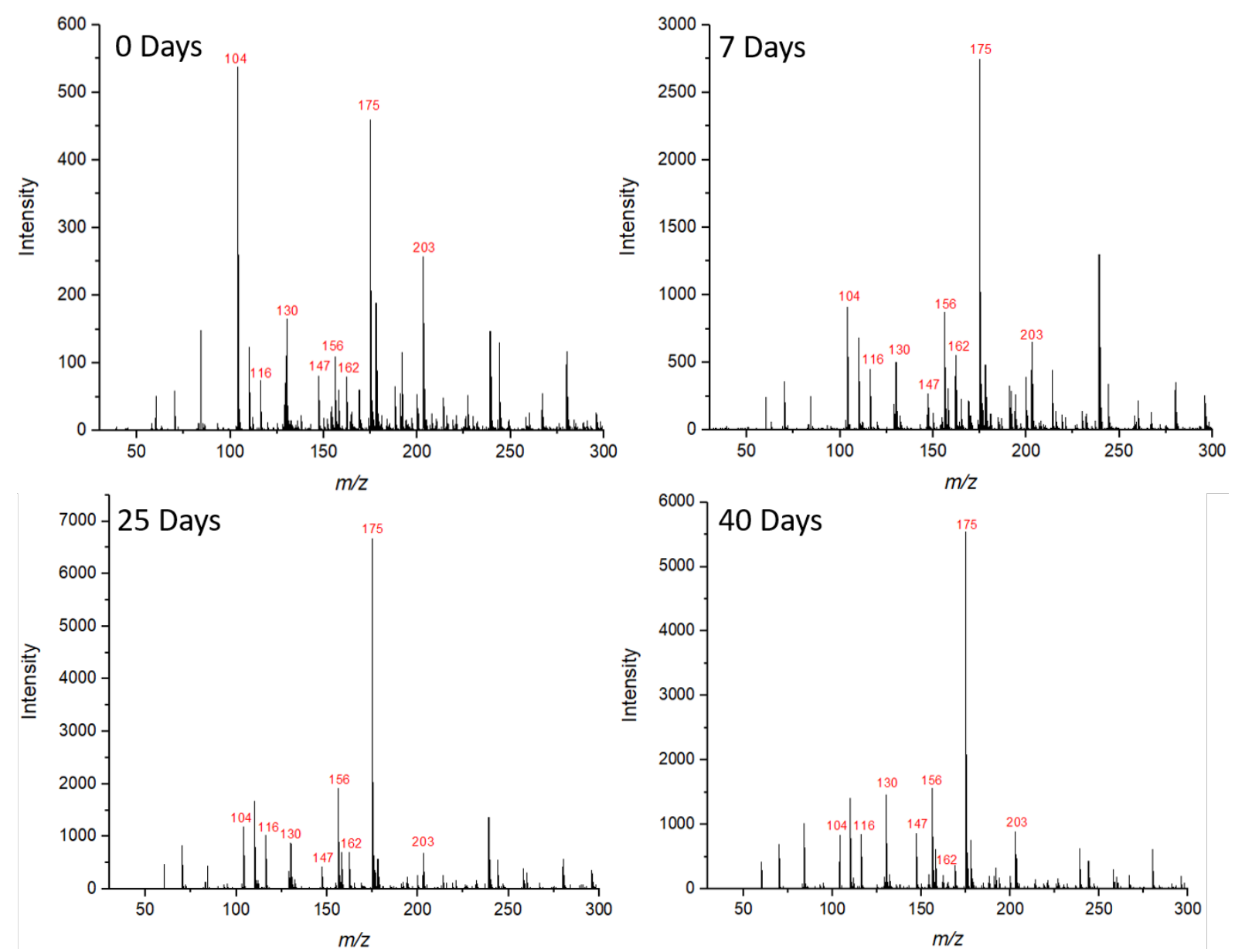

Figure 2. Mass spectra of semen sampled from a glass slide, aged over a period of 40 days.

In the development of techniques for body fluid analysis in criminal investigations, biological samples may be encountered weeks, months or even years after deposition. It is therefore essential to confirm the capability of new techniques in the detection of aged samples. The chemical profile of human semen after a period of 40 days remained largely unchanged (Figure 2). Almost all of the identified components, particularly choline, histidine, arginine and spermine, were still readily detected after 40 days of ageing. This demonstrates the possibility of confirming the presence of semen at least 40 days after deposition. The abundance of characteristic compounds remaining in the aged sample and slow degradation or loss of these compounds suggests the technique may be capable of identification of even older samples, though further investigations would be required to confirm this. However, in the analysis of aged semen on fabric, the spectrum was dominated by ions originating from the solvent and background (Figure S4). Although analytes originating from the semen could be detected, such as choline, glutamine, arginine and spermine, the response was weak and in some cases no analyte ions were observed, only background ions. This demonstrates the effect of different 
matrices on the effectiveness of sfPESI-MS. The relative standard deviation of the method was approximately $20 \%$ (based on the abundance of major ions throughout repeat analyses, $n=6$ ). Despite the perseverance of components prevalent in the semen samples, several changes were observed, over time as the samples were exposed to the ambient environment. As illustrated in Figure 2, by the 40-day timepoint, many of the low abundance components have been reduced or, in some cases, completely lost. The relative intensity of the most abundant components also altered over time, with choline, initially the most abundant ion detected, decreasing, and arginine later becoming the most abundant component. In summary, sfPESI-MS was demonstrated to be a powerful new technique in semen analysis. However, in the context of a forensic investigation, it is possible that semen may be associated with evidence such as condoms and sexual lubricants, which can also provide useful chemical information.

\subsection{Condom Analysis}

As offenders become more knowledgeable about DNA evidence and how biological material can be used to link perpetrators to crime scenes, the use of condoms in sexual assaults has increased.[20] In the absence of biological evidence such as semen, trace evidence of condoms or sexual lubricants may be essential physical evidence in indicating the sequence of events, corroborating the statement of the victim, and demonstrating a degree of premeditation.

Previous research has indicated that it may be possible to differentiate between condom brands based on the chemical composition of the condom and lubricants. Musah, et al utilized Direct Analysis in Real Time mass spectrometry (DART-MS) to directly analyse condoms, demonstrating the possibility of detecting components specific to certain types and brand of condom.[21] Similarly, DART-MS has also been used in the analysis of sexual lubricants, demonstrating the chemical differences between 33 different lubricants.[22] Other mass spectrometric techniques have also been applied to the analysis of condoms and lubricants. Bradshaw et al applied MALDI-MSI to the mapping of condom lubricants in fingermarks, demonstrating the ability to detect lubricants in fingerprints weeks after deposition and differentiate between lubricants from different condom brands.[23][24] Furthermore, van Helmond et al demonstrated the classification of condom lubricants in treated fingerprints using DESI-MS.[25] Furthermore, chemometric strategies have been developed capable of differentiating between condom brands based on their chemical profiles.[26], [27] In light of this, it is therefore important to demonstrate that any techniques developed for the direct 
analysis of suspected semen, would also be capable of detecting components pertinent to condoms and lubricants, in order to develop a technique more widely applicable to sexual assault evidence analysis.

In this study, a series of eight condom types (full details can be found Table S1 in the supplementary information) were subjected to direct analysis by the sfPESI probe for the purpose of confirming that condom-specific components could be detected and to ascertain the chemical differences between different condom brands. Each condom produced a unique mass spectral profile (Figure 3), though a number of analogous components could be detected in multiple samples. 

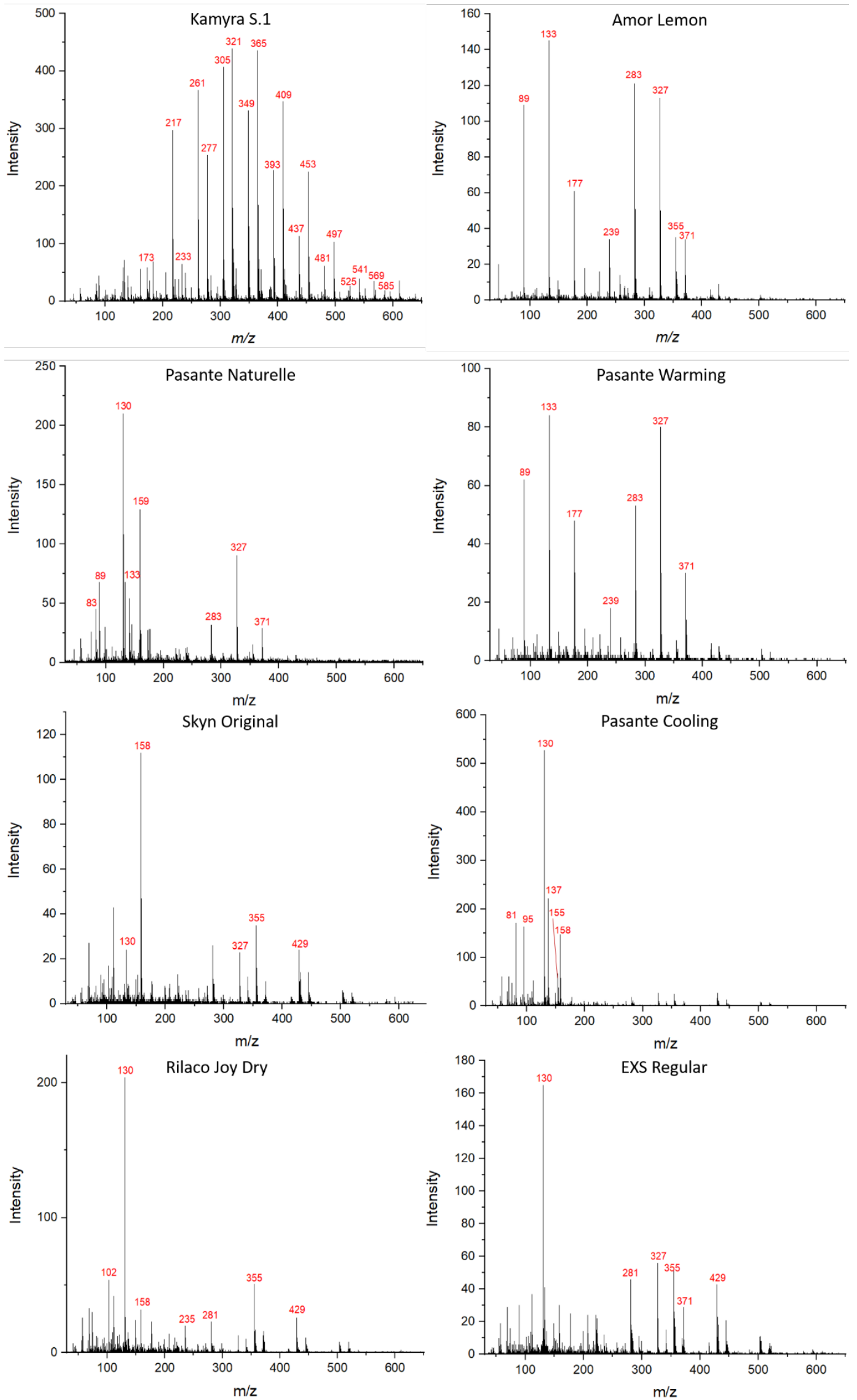

Figure 3. Mass spectra of condom brands acquired using sheath-flow probe electrospray ionisation mass spectrometry. 
A prominent ion detected at $\mathrm{m} / \mathrm{z} 130$ could be detected in the majority of condom samples. Based on MS/MS experiments producing fragments at $\mathrm{m} / \mathrm{z} 57$ and 74 , this was identified as the $[\mathrm{M}+\mathrm{H}]^{+}$ion of octylamine, a component frequently encountered in the analysis of condoms. Also detected in many of the samples were the $m / z 327,355$ and 371 ions, corresponding to protonated caprylic triglyceride, caprylic/capric triglyceride and the polydimethylsiloxane $\mathrm{PDMS}_{5}$, all known components in lubricants, such as those used on condoms.[28] A series of ions detected at $m / z$ 89, 133, 177, 239, 327 and 371 were prominent in the Amor Lemon and Pasante Warming brands, ions commonly associated with polyethylene glycol oligomers.[29], [30]

Despite these similarities, a number of constituents were detected more specific to the type of condom analysed. The Kamyra S.1 condom exhibited a particularly distinctive mass spectrum in comparison to the other brands, exhibiting a characteristic distribution of ions different by 44 mass units. This pattern, previously detected in the analysis of condoms and lubricants,[31] has been identified as a polyethylene glycol (PEG) series, observed in a trend of $[\mathrm{M}+\mathrm{Na}]^{+}$ions at $m / z 173,217,261,305,349,393,437,481,525$ and 569 , and $[\mathrm{M}+\mathrm{K}]^{+}$ions at $m / z$ 233, 277, 321, 365, 409, 453, 497, 541 and 585.[32] The Kamyra condom was also advertised as containing a spermicide, specifically nonoxynol-9, one of the most widely used active ingredients in spermicidal products (though now controlled by some countries due to health concerns). This component typically appears as a distribution of ions observed at $\mathrm{m} / \mathrm{z}$ $397,441,485,529,573,617,661,705$, and 749.[21] In this study, only small peaks were detected at $\mathrm{m} / \mathrm{z} 397$ and 441, respectively, corresponding to the N4 and N5 nonoxynol-9 components. Those at a higher mass could not be detected, potentially due to ion suppression caused by the prominent PEG ions dominating the mass spectrum.

The Pasante Cooling condom is specifically designed to induce a 'cooling effect' on the user. These effects are often achieved by the addition of mint-associated compound such as menthol, carvone or other similar substances to the lubricants applied to the sheath. In this study, an ion was detected at $m / z 155$, which likely corresponds to the $[\mathrm{M}+\mathrm{H}]^{+}$ion of menthone, a component frequently used in mint flavourings. Furthermore, ions were also detected at $\mathrm{m} / \mathrm{z}$ 81 and 137. Both of these are often associated with the presence of terpenes such as menthone, with $\mathrm{m} / \mathrm{z} 81$ being a common monoterpene fragment and $\mathrm{m} / \mathrm{z} 137$ attributed to the loss of water from the protonated monoterpene.[33]

One latex-free brand was used in this study. The Skyn Original condom primarily differed from its latex counterparts in the presence of an abundant ion at $m / z 158$. Based on MS/MS 
experiments producing fragment ions at $\mathrm{m} / z 57$ and 102 , this has been identified as the $[\mathrm{M}+\mathrm{H}]^{+}$ form of N,N-dibutylformamide. This compound was also detected in the lubricant-free Rilaco Joy brand, along with ions at $\mathrm{m} / \mathrm{z} 102$ and 130, pertaining to protonated N,N-methylmorpholine and octylamine respectively. All of these are chemicals known to be found in condoms.[27] The Pasante warming and Amor lemon-flavoured condoms produced similar mass spectra. Interestingly, components specific to these condom types that may have been used to produce the lemon flavour and warming effect could not be detected. For instance, methyl salicylate and menthol are often used in sexual lubricants to produce warming effects,[34] but these components were not observed. However, without access to the ingredients used in the condoms, it is difficult to confirm which chemicals may have been used during production.

\section{Conclusions}

In conclusion, sheath-flow probe electrospray ionisation mass spectrometry has been applied to the direct analysis of evidence relevant to sexual assault investigations, necessitating no sample preparation and enabling sample analysis in less than 10 seconds. For the first time, ambient ionisation was applied to the forensic analysis of semen, demonstrating the capability of detecting the presence of both fresh and dried semen (up to 40 days in age) from surfaces of various materials and porosities. A range of metabolites could be detected and, by coupling the sfPESI probe with tandem mass spectrometry, confirmatory compound identification could be achieved. Not only was it possible to detect components that could be used to confirm the presence of semen, such as spermine and choline, but also study chemical changes occurring in semen over time. Finally, the probe was readily applied to the analysis of different condom brands, demonstrating the possibility of detecting components that could be used to indicate condom use by a perpetrator, as well as differentiating between condom brands based on differences in the chemical composition of both the condom sheath and the lubricants used. The sfPESI probes used in this work are cheap, disposable items that can easily be used to sample in the field or be transported to the lab for rapid analysis with sample run times of $<10$ seconds, confirming the presence of semen on contaminated sample surfaces. This study has demonstrated a powerful new technique for the analysis of semen and other sexual assault evidence to facilitate faster and more efficient forensic analysis. 


\section{Acknowledgements}

The authors gratefully acknowledge funding from the Engineering and Physical Sciences Research Council (EP/N509516/1).

\section{Conflict of Interest Statement}

The authors declare there are no conflicts of interest.

\section{References}

[1] Office for National Statistics, "Crime in England and Wales: Year Ending December 2018," 2018.

[2] Home Office and Ministry of Justice, "An Overview of Sexual Offending in England and Wales," 2013.

[3] K. Virkler and I. K. Lednev, "Analysis of body fluids for forensic purposes: From laboratory testing to non-destructive rapid confirmatory identification at a crime scene," Forensic Sci. Int., vol. 188, no. 1-3, pp. 1-17, 2009, doi: 10.1016/j.forsciint.2009.02.013.

[4] A. Jamieson and S. Bader, A guide to forensic DNA profiling. Chichester: John Wiley \& Sons, Ltd., 2016.

[5] J. Old, B. A. Schweers, P. W. Boonlayangoor, B. Fischer, K. W. P. Miller, and K. Reich, "Developmental Validation of RSID" ${ }^{\mathrm{TM}}$-Semen: A Lateral Flow Immunochromatographic Strip Test for the Forensic Detection of Human Semen," $J$. Forensic Sci., vol. 57, no. 2, pp. 489-499, Mar. 2012, doi: 10.1111/j.15564029.2011.01968.x.

[6] K. Van Steendam, M. De Ceuleneer, M. Dhaenens, D. Van Hoofstat, and D. Deforce, "Mass spectrometry-based proteomics as a tool to identify biological matrices in forensic science," Int. J. Legal Med., vol. 127, no. 2, pp. 287-298, 2013, doi: 10.1007/s00414-012-0747-X.

[7] H. Yang, B. Zhou, H. Deng, M. Prinz, and D. Siegel, "Body fluid identification by mass spectrometry," Int. J. Legal Med., vol. 127, no. 6, pp. 1065-1077, Nov. 2013, doi: 10.1007/s00414-013-0848-1.

[8] X. Chen, C. Hu, J. Dai, and L. Chen, "Metabolomics Analysis of Seminal Plasma in Infertile Males with Kidney-Yang Deficiency: A Preliminary Study," Evidence-Based Complement. Altern. Med., vol. 2015, pp. 1-8, 2015, doi: 10.1155/2015/892930.

[9] X. Zhang, R. Diao, X. Zhu, Z. Li, and Z. Cai, "Metabolic characterization of asthenozoospermia using nontargeted seminal plasma metabolomics," Clin. Chim. Acta, vol. 450, pp. 254-261, Oct. 2015, doi: 10.1016/j.cca.2015.09.001.

[10] J. Wang et al., "Proteomic analysis of seminal plasma from asthenozoospermia patients reveals proteins that affect oxidative stress responses and semen quality," Asian J. Androl., vol. 11, no. 4, pp. 484-491, Jul. 2009, doi: 10.1038/aja.2009.26.

[11] V. Longo et al., "HS-SPME-GC-MS metabolomics approach for sperm quality 
evaluation by semen volatile organic compounds (VOCs) analysis," Biomed. Phys. Eng. Express, vol. 5, no. 1, p. 015006, Nov. 2018, doi: 10.1088/2057-1976/aaeb07.

[12] S. Rankin-Turner, M. A. Turner, P. F. Kelly, R. S. P. King, and J. Reynolds, "Transforming Presumptive Forensic Testing: In-situ Identification and Age Estimation of Human Bodily Fluids," Chem. Sci., vol. In press, 2019, doi: 10.1039/C8SC04133D.

[13] M. J. Bailey et al., "Analysis of urine, oral fluid and fingerprints by liquid extraction surface analysis coupled to high resolution MS and MS/MS - opportunities for forensic and biomedical science," Anal. Methods, vol. 8, no. 16, pp. 3373-3382, 2016, doi: 10.1039/C6AY00782A.

[14] J. M. Wiseman, C. a Evans, C. L. Bowen, and J. H. Kennedy, "Direct analysis of dried blood spots utilizing desorption electrospray ionization (DESI) mass spectrometry.," Analyst, vol. 135, no. 4, pp. 720-725, 2010, doi: 10.1039/b922329k.

[15] M. O. Rahman et al., "Development of sheath-flow probe electrospray ionization (SFPESI)," J. Mass Spectrom., vol. 48, no. 7, pp. 823-829, Jul. 2013, doi: 10.1002/jms.3226.

[16] D. T. Usmanov et al., "Remote sampling mass spectrometry for dry samples: Sheathflow probe electrospray ionization (PESI) using a gel-loading tip inserted with an acupuncture needle," Rapid Commun. Mass Spectrom., vol. 32, no. 5, pp. 407-413, Mar. 2018, doi: 10.1002/rcm.8045.

[17] S. Rankin-Turner, S. Ninomiya, J. C. Reynolds, and K. Hiraoka, "Sheath-flow probe electrospray ionization (sfPESI) mass spectrometry for the rapid forensic analysis of human body fluids," Anal. Methods, 2019, doi: 10.1039/C9AY00698B.

[18] D. S. Wishart et al., "HMDB 4.0: the human metabolome database for 2018," Nucleic Acids Res., vol. 46, no. D1, pp. D608-D617, Jan. 2018, doi: 10.1093/nar/gkx1089.

[19] P. Hardinge, J. Allard, A. Wain, and S. Watson, "Optimisation of choline testing using Florence Iodine reagent, including comparative sensitivity and specificity with PSA and AP tests," Sci. Justice, vol. 53, no. 1, pp. 34-40, Mar. 2013, doi: 10.1016/j.scijus.2012.03.004.

[20] E. N. O’Neal, S. H. Decker, C. Spohn, and K. Tellis, "Condom use during sexual assault," J. Forensic Leg. Med., vol. 20, no. 6, pp. 605-609, Aug. 2013, doi: 10.1016/j.jflm.2013.03.023.

[21] R. A. Musah, R. B. Cody, A. J. Dane, A. L. Vuong, and J. R. E. Shepard, "Direct analysis in real time mass spectrometry for analysis of sexual assault evidence," Rapid Commun. Mass Spectrom., vol. 26, no. 9, pp. 1039-1046, 2012, doi: 10.1002/rcm.6198.

[22] C. Bridge and M. Marić, "Temperature-Dependent DART-MS Analysis of Sexual Lubricants to Increase Accurate Associations," J. Am. Soc. Mass Spectrom., Mar. 2019, doi: 10.1007/s13361-019-02158-x.

[23] R. Bradshaw et al., "Spectroscopic imaging based approach for condom identification in condom contaminated fingermarks," Analyst, vol. 138, no. 9, pp. 2546-2557, 2013, doi: 10.1039/c3an00195d.

[24] R. Bradshaw, R. Wolstenholme, R. D. Blackledge, M. R. Clench, L. S. Ferguson, and S. Francese, "A novel matrix-assisted laser desorption/ionisation mass spectrometry imaging based methodology for the identification of sexual assault suspects," Rapid 
Commun. Mass Spectrom., vol. 25, no. 3, pp. 415-422, Feb. 2011, doi: $10.1002 / \mathrm{rcm} .4858$.

[25] W. van Helmond, M. P. V. Begieneman, R. Kniest, and M. de Puit, "Classification of condom lubricants in cyanoacrylate treated fingerprints by desorption electrospray ionization mass spectrometry," Forensic Sci. Int., vol. 305, p. 110005, Dec. 2019, doi: 10.1016/j.forsciint.2019.110005.

[26] A. M. Coon, S. Beyramysoltan, and R. A. Musah, "A chemometric strategy for forensic analysis of condom residues: Identification and marker profiling of condom brands from direct analysis in real time-high resolution mass spectrometric chemical signatures," Talanta, vol. 194, no. July 2018, pp. 563-575, Mar. 2019, doi: 10.1016/j.talanta.2018.09.101.

[27] M. F. Mirabelli, D. R. Ifa, G. Sindona, and A. Tagarelli, "Analysis of sexual assault evidence: statistical classification of condoms by ambient mass spectrometry," J. Mass Spectrom., vol. 50, no. 5, pp. 749-755, May 2015, doi: 10.1002/jms.3584.

[28] M. Maric, L. Harvey, M. Tomcsak, A. Solano, and C. Bridge, "Chemical discrimination of lubricant marketing types using direct analysis in real time time-offlight mass spectrometry," Rapid Commun. Mass Spectrom., vol. 31, no. 12, pp. 1014 1022, Jun. 2017, doi: 10.1002/rcm.7876.

[29] A. Onigbinde, G. Nicol, and B. Munson, "Gas Chromatography/Mass Spectrometry of Polyethylene Glycol Oligomers," Eur. J. Mass Spectrom., vol. 7, no. 3, pp. 279-291, Jun. 2001, doi: 10.1255/ejms.438.

[30] S.-T. Lai, "Characterization and molecular weight determination of water-soluble polyethylene glycol oligomers using open-tubing liquid chromatography - mass spectrometry," J. Chromatogr. A, vol. 363, no. 2, pp. 444-447, Jan. 1986, doi: 10.1016/S0021-9673(01)83772-9.

[31] P. Maynard, K. Allwell, C. Roux, M. Dawson, and D. Royds, "A protocol for the forensic analysis of condom and personal lubricants found in sexual assault cases," Forensic Sci. Int., vol. 124, no. 2-3, pp. 140-156, Dec. 2001, doi: 10.1016/S03790738(01)00588-6.

[32] D. R. Ifa, L. M. Gumaelius, L. S. Eberlin, N. E. Manicke, and R. G. Cooks, "Forensic analysis of inks by imaging desorption electrospray ionization (DESI) mass spectrometry," Analyst, vol. 132, no. 5, p. 461, 2007, doi: 10.1039/b700236j.

[33] L. M. Heaney et al., "Real-time monitoring of exhaled volatiles using atmospheric pressure chemical ionization on a compact mass spectrometer," Bioanalysis, vol. 8, no. 13, pp. 1325-1336, 2016, doi: 10.4155/bio-2016-0045.

[34] S. E. Spencer, S. Y. Kim, S. B. Kim, and K. A. Schug, "Matrix-assisted laser desorption/ionization-time of flight-mass spectrometry profiling of trace constituents of condom lubricants in the presence of biological fluids," Forensic Sci. Int., vol. 207, no. 1-3, pp. 19-26, Apr. 2011, doi: 10.1016/j.forsciint.2010.08.010. 